\title{
关于蔬果农药残留现象的实践调研
}

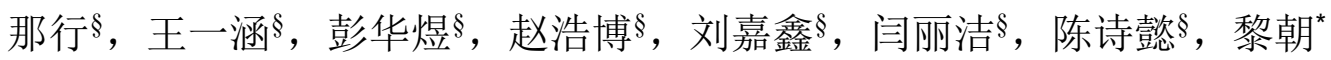

厦门大学化学化工学院, 化学国家级实验教学示范中心(厦门大学), 福建 厦门 361005

摘要: 厦门大学化学化工学院 “一点一滴” 实践队结合所学专业知识, 针对果蔬农药残留现象进行了实践调研。通 过对不同背景市民进行的调查访问, 了解到他们对农药残留的认识及日常采用的去除办法存在一定疑惑。在大量文 献调研的基础上, 总结目前农药残留的检测手段, 从中筛选并优化适合本次实践活动的测定方法。对部分去除农药 残留的方法进行实验检验, 根据测定结果给出相应的建议。本次实践活动将所学专业知识运用于实际, 培养和提高 了学生的实践能力和综合素质。

关键词: 食品安全; 农药残留; 实践调研

中图分类号: G64; O6

\section{Practical Investigation on Pesticide Residues in Fruits and Vegetables}

\author{
Xing $\mathrm{Na}{ }^{\S}$, Yihan Wang ${ }^{\S}$, Huayu Peng ${ }^{\S}$, Haobo Zhao ${ }^{\S}$, Jiaxin Liu ${ }^{\S}$, Lijie Yan ${ }^{\S}$, Shiyi Chen ${ }^{\S}$, Zhao Li * \\ National Demonstration Center for Experimental Chemistry Education (Xiamen University), College of Chemistry and \\ Chemical Engineering, Xiamen University, Xiamen 361005, Fujian Province, P. R. China.
}

\begin{abstract}
The "Drops" practice team from School of Chemistry and Chemical Engineering of Xiamen University carried out a practical investigation on the phenomenon of pesticide residues in fruits and vegetables with the combination of professional knowledge. Through the investigation and interview of citizens from different backgrounds, it is found that there are some doubts about their understanding of pesticide residues and the removal methods used daily. On the basis of a large number of literature research, the current detection methods of pesticide residues were summarized, and the methods suitable for this practice were selected and optimized. The method of partial removal of agricultural residues was tested experimentally and some suggestions were given according to the results. This practice will apply professional knowledge to the practice, training and improving the students' practical ability and comprehensive quality.
\end{abstract}

Key Words: Food safety; Pesticide residues; Practice research

食品安全问题一直是全社会关心的焦点问题。在大量食品安全话题刷屏的当今社会, 甚至有些 人开始调㑆: 中国到底容不容得下一张 “安静的” 餐桌? 这句话显然言过其实, 在我们看来, 当今 市民应该科学认识农产品农药残留问题, 既需要了解农药残留的具体危害及相对有效的去除办法, 也需要具备一定辨识谣言的能力，不要谈虎色变。

针对农药残留及相关问题, 厦门大学化学化工学院的 “一点一滴” 实践队针对不同背景的人群 采取了现场采访、调查问卷等多样的调查形式, 并且访问了专业生产食品安全检测仪、食品安全快

收稿: 2020-03-03; 录用: 2020-03-19; 网络发表: 2020-04-28

*通讯作者, Email: lizhao@xmu.edu.cn

$\S 2018$ 级本科生 
速检测箱的厦门市海荭兴仪器股份有限公司, 对目前食品安全检测、农残快速检测等方面的市场需 求和推广问题进行了调研。最后对去除农药残留的部分方法进行实验验证，给出相应建议。

\section{1 社会调研}

首先, 我们应该要清楚认识农药残留的概念。根据中华人民共和国农业农村部给出的解释, 农 药残留是指使用农药后残留于农产品、食品及环境中的微量农药及其有毒代谢物和杂质。农药残留 其实是农产品施药之后的正常现象，难以避免。

我国是一个农业大国, 农产品是食品的主要来源, 使用农药控制病虫草害从而确保粮食丰产是 必要的技术措施。像容忍药物的副作用一样, 我们也应该理解和接受经过农产品生长代谢消减后的 痕量农药残留。对农药残留 “零容忍” 的说法很不科学, 也很不理智。

我们需要明白, 现在的分析手段, 能做到检测出痕量的农药残留。只要这些农药残留没有超过 一定的剂量水平, 就是安全的, 就不会对人体健康产生危害。我国标准 GB2763-2019 规定了 356 种 (类)食品中 483 种农药共 7107 项最大残留限量。根据农业农村部组织的国家农产品质量安全例行监 测(风险监测)结果表明, 近年来我国农产品质量抽检总体合格率已稳定达到 97\%以上, 所以我国农 产品消费安全是有保障的。

虽然说痕量的农药残留基本对健康没有影响, 但是一旦农药残留过量将会对身体造成一定程度 的危害。我们无法通过外观判断农残超标的蔬菜和水果, 所以仍然需要具备对农药残留基础的认知 以及处理方法。我们计划通过问卷调查、采访以及企业调研等方式来了解社会各个层面对于农药残 留现象的了解。

\section{1 调查问卷及采访分析}

依据生活经验, 我们按照是否为在校学生对调查群体进行分类, 再根据其买菜的频率细致划分。 这样分类的好处在于我们可以很清晰地对比出不同背景人群的选择, 便于之后的分析。在此需要说 明的是, 由于本次实践活动受时间、经费等方面的限制, 我们的调查问卷样本量有限。以下数据和 分析结论都来源于我们本次问卷调查，有可能不能够全面反映社会真实情况。

我们选取了日常生活中最常见的事件，如：哪些购买点比较安全可信？是否会选择绿色食品？ 清洗果蔬时是否会使用某些方法去除农药残留等等, 共设置了 13 个问题。对不同背景的人设问方式 不同，是希望得到最真实、准确的答案。

本次调查共回收问卷 347 份, 其中在校学生 221 份, 非在校学生 126 份。随后, 我们选取部分 人依据调查结果进一步现场采访分析, 以获得更为全面立体的答案。但由于我们的样本总量及覆盖 面有限, 所以在此以介绍问题设置和探究形式为主。

首先, 我们发现在受访者中, 经常买菜和不经常买菜的人对于买菜地点的选择不尽相同, 如 图 1(a)所示。对于经常买菜的人来说, 菜市场和大型超市是他们的首选; 对于不经常买菜的人来 说, 精品超市则是最佳的购买点。从身份分析, 如图 1(b)所示, 受访学生和其他人群之间没有特别 大的差异, 仅仅是学生选择菜市场的人数较少。为了解造成经常买菜和不经常买菜受访者差异的原 因，我们对部分受访者做了进一步的采访。

采访后我们得知, 经常买菜的受访者选择菜市场的主要原因是菜市场的菜看起来更为新鲜、价 格更为实惠。至于他们为什么不选择网购和精品超市, 大部分受访者的答案是菜价较为昂贵或无法 判断蔬果是否新鲜。而不常买菜的受访者选择精品市场是因为这些超市会售卖带有绿色食品标识的 蔬果，以及进口蔬果。他们认为这些产品的安全性更有保障。

从 1990 年起, 我国便开始使用绿色食品标志。绿色食品的覆盖范畴非常广, 不仅有蔬果、粮 食, 还有禽畜类、水产类、饮料类等产品。

参考 1993 年颁布的绿色食品标志管理办法, 绿色食品是指产自优良生态环境、按照绿色食品标 准生产、实行全程质量控制并获得绿色食品标志使用权的安全、优质食用农产品及相关产品, 根据 不同要求, 分为 $\mathrm{A} 、 \mathrm{AA}$ 两个等级。 
(a)

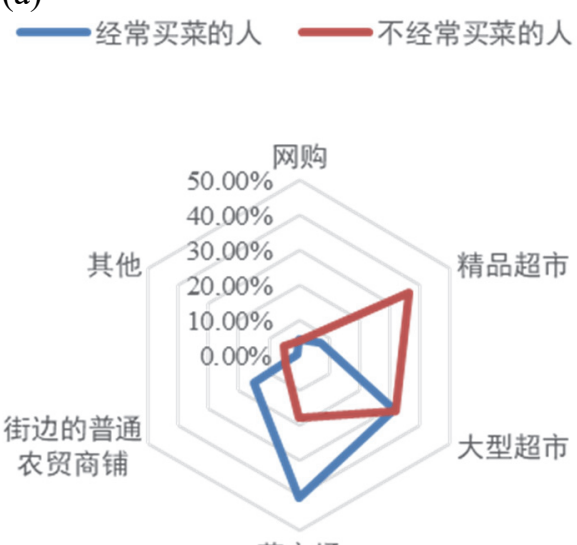

菜市场 (b)

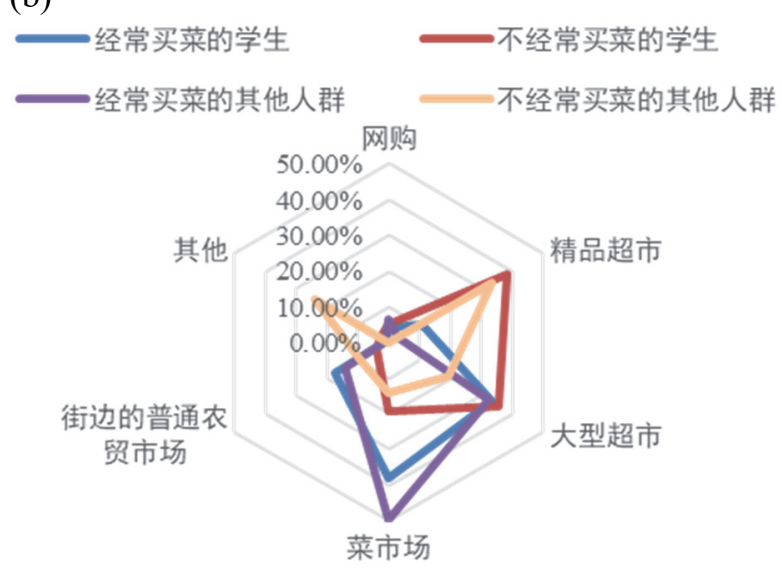

图 1 (a) 受访者的买菜频率和购买蔬果地点选择的关系; (b) 不同背景受访者的买菜频次和购买蔬果地点的关系

在我们的调查中, 较多的受访者不是很清楚我国绿色蔬菜等级分为 A、AA 两级, 如图 2(a)所 示。根据进一步调查发现, 受访者普遍知道绿色食品的基本定义, 但是不清楚其分为两级并具有不 同的标识。事实上 $\mathrm{A}$ 级绿色食品是允许使用限定的化学合成物质的, 只有 $\mathrm{AA}$ 级绿色食品才是不允 许使用任何有害化学合成物质。二者标志的区别在于: A 级标志为绿底白字, AA 级标志为白底绿 字。

此外还有一类不使用化学农药、化肥、化学防腐剂等合成物质, 也不用基因工程生物及其产物 的农产品, 称为有机食品。进一步调查的结果表示, 买菜的受访者中有 $30 \%$ 认为不需要特意购买有 绿色食品标识的蔬菜水果。我们进一步再对人群进行分类, 我们发现受访者中, 经常买菜的其他人 群要比学生更愿意购买有绿色食品标识的蔬菜, 如图 2(b)所示。

(a)

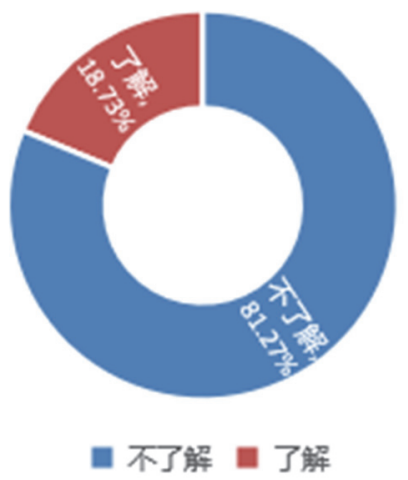

(b)

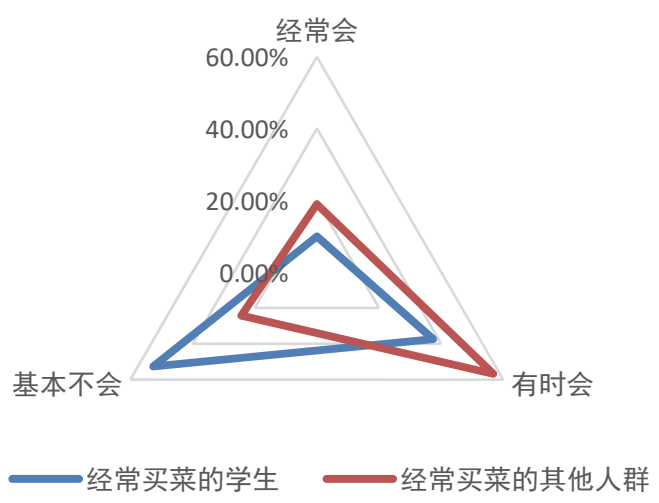

图 2 (a) 受访者是否了解两种不同的绿色食品标识; (b) 不同背景受访者购买绿色食品标志蔬菜的倾向

另外我们调查了受访者自认为农药残留最高的蔬菜水果, 结果如图 3(a)、3(b)所示。被认为农药 残留较高的蔬菜水果有部分统一的特性: (1) 直接食用, 无需去皮; (2) 表面多皱褶, 比表面积大; (3) 叶片较大。这些特性确实更容易致使农药残留。

为了验证受访者的猜想, 我们找到了美国环境工作组(EWG)于 2019 年公布的农药残留相对较高 的蔬菜水果, 如表 1 所示。 
(a)

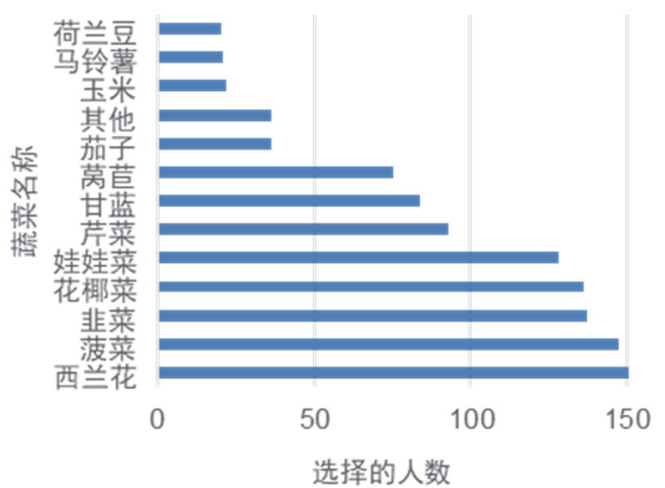

(b)

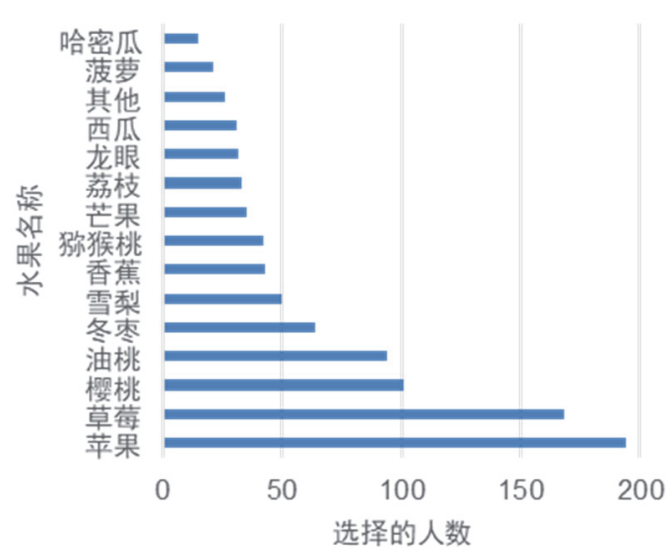

图 3 (a) 受访者心中农药残留最高的蔬菜排序图; (b) 受访者心中农药残留最高的水果排序

表 12019 年 EWG 给出的水果、蔬菜农药残留排位

\begin{tabular}{cc||cc}
\hline 水果名称 & 排名(总排名 $\left.{ }^{*}\right)$ & 蔬菜名称 & 排名(总排名) \\
\hline 草莓(Strawberries) & $1(1)$ & 菠菜(Spinach) & $1(2)$ \\
油桃(Nectarines) & $2(4)$ & 羽衣甘蓝(Kale) & $2(3)$ \\
苹果(Apples) & $3(5)$ & 芹菜(Celery) & $3(11)$ \\
葡萄(Grapes) & $4(6)$ & 土豆(Potatoes) & $4(12)$ \\
水蜜桃(Peaches) & $5(7)$ & 甜椒(Sweet Bell Peppers) & $5(13)$ \\
樱桃(Cherries) & $6(8)$ & 生菜(Lettuce) & $6(15)$ \\
梨(Pears) & $7(9)$ & 黄瓜(Cucumbers) \\
番茄(Tomatoes) & $8(10)$ & 红辣椒(Hot Peppers) & $7(16)$ \\
圣女果(Cherry Tomatoes) & $9(14)$ & 四季豆(Green Beans) & $8(18)$ \\
蓝莓(Blueberries) & $10(17)$ & 冬瓜(Winter Squashes) & $9(20)$ \\
李子(Plums) & $11(19)$ & 踠豆(Snap Beans) & $10(24)$ \\
橙子(Tangerines) & $12(21)$ & 胡萝卜(Carrots) & $11(25)$ \\
\hline
\end{tabular}

*总排名为 $\mathrm{EWG}$ 公布的 2019 年蔬菜水果农药残留 TOP50 数据

对比数据我们发现, 受访者对于可能含过量农药残留的蔬菜水果判断还是比较准确的, 除了部 分蔬菜水果排位在 EWG 给出的数据中排位较低, 如蔬菜中西兰花位列总排名第 35 , 花椰菜位于 37 。 水果中香蕉位于 30 , 猕猴桃位于 39 。甘蓝和马铃薯则相对排位较高, 分别为第 3 和第 12 。

对于处理农药残留的方法, 我们也进行了调查, 结果如图 4 所示。选择会处理农药残留的受访 者中, 大部分会选用盐水、蔬果洗涤剂这类具备杀菌功能的洗液。在现场采访中, 还有些受访者给 出很多别出心裁的点子, 例如使用环保酵素、淀粉加水等等方法。

在关于造成食物中毒原因的调查中(图 5), 近 3 成的受访者选择了农药残留, 仅次于非农药的非 法化学添加剂。这说明农药残留在这些受访者心中是造成食物中毒较为可能的原因之一, 所以选择 合适的方法预防是很有必要的。

从我们的调查数据来看, 有部分受访者使用的清洗方法缺乏科学性, 有时甚至有可能适得其反。 例如, 对于某些碱性稳定的农药, 用小苏打水清洗反而会导致农药残留更稳定, 不易清除。这让我 们认识到, 对于农药残留的相关科普, 以及针对清洗农药残留的方式探究是十分有意义且十分重要 的。 


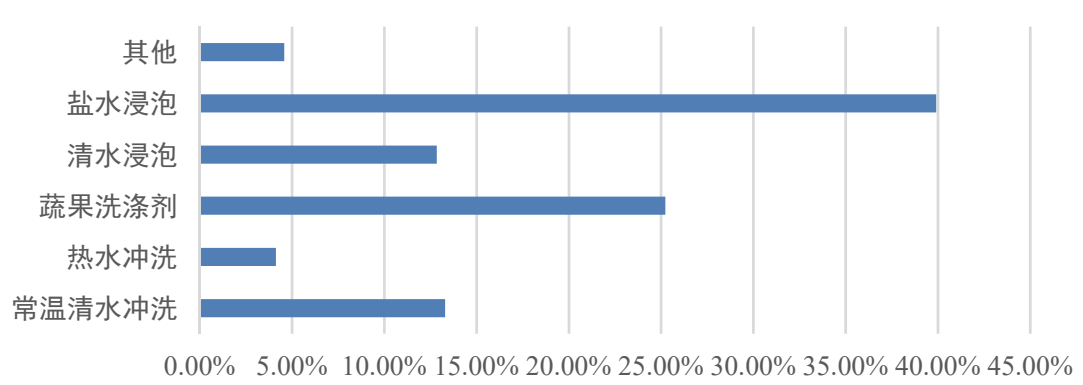

图 4 市民认为清除农药残留的有效处理办法

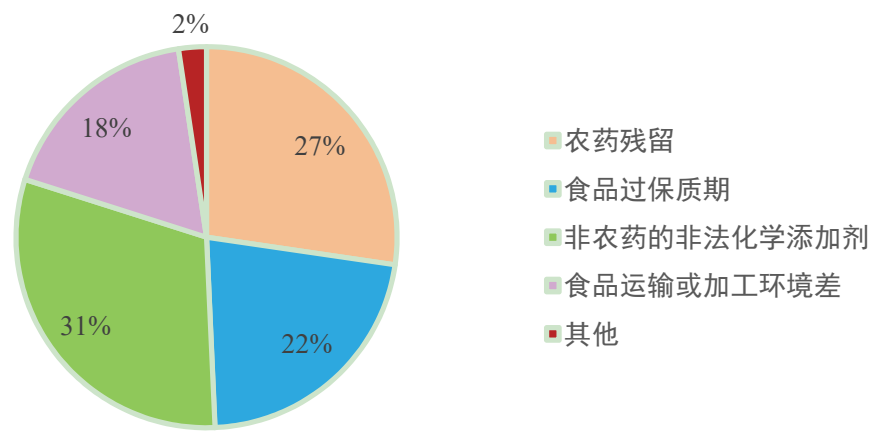

图 5 市民认为造成食物中毒的原因

最后, 我们在问卷中提及, 如果有十分廉价便捷且能快速检测农药残留的试纸, 受访者是否愿 意购买并使用, 或推荐家人使用。近 9 成受访者选择了愿意。这说明, 在价格合理、方便快捷的情 况下，受访者非常愿意选择使用农药残留检测试纸。

这里还需要做出一定解释的是, 检测农药残留的方法和标准每个国家不尽相同, 选用别国的标 准来判断是否存在安全问题, 或用一国标准否定别国的标准都是非常不恰当的行为。尽管制定残留 标准的主要目的是为了确保食品农产品质量安全, 但根据农产品质量检查司的报告, 现在不少国家 将农药残留作为农产品国际贸易的技术壁垒, 甚至用作政治筹码。我国按照国际上通用的风险评估 技术和方法, 以考虑最大可能的风险为原则, 制定了农药残留限量国家标准, 是完全能够确保国民 健康安全的。

\section{2 厦门海荭兴仪器股份有限公司调研}

2019 年 7 月 5 日, 我们实践队前往厦门海荭兴仪器股份有限公司进行参观和采访。实践队就农 药残留检测仪器做了详细的了解, 参观了公司的研发区及其实验室。

我们了解到, 面对每天需要进行的大批量大范围的食品质量检测, 目前主要存在以下几方面的 难点: (1) 实验室设备数量有限; (2) 实验室样品检测数量有限; (3) 实验室样品检测周期长; (4) 实 验室样品检测费用高等。随着社会发展, 一方面食品检测的需求量一再提高, 另一方面许多价廉、 普遍食用但容易出现问题的食品, 如菜市场销售的蔬果, 需要花费其本身价格成百上千倍的检测费 用由实验室来进行检测。以这样的方法全面保障食用者的食品安全是不现实的, 而快速、低成本的 检测则是一种可行的保障手段。

海荭兴仪器股份有限公司的仪器从不同角度、以不同精度检测农药及兽药残留, 其原理包括紫 外可见分光光度法、核磁共振法、表面增强拉曼光谱法、酶指示法等等。其中紫外可见分光光度法 由于其价格便宜、精度较高而被广泛使用; 而酶指示法则具有快速检测的基础, 在快检试纸的应用 和推广方面具有非常大的潜力。 
此外, 我们还了解了公司的发展历程, 明白了科学技术转化为生产力的途径, 以及之间的艰辛 与付出。在亲自尝试使用仪器和试纸时, 我们感受到快速检验的便捷性与重要性。

\section{2 文献调研}

实践队成员首先以 “有机磷农药” “农药残留检测方法” 等为关键词, 利用百度学术、Google 学术、中国知网等搜索引擎和数据库, 查阅了一批中外文献, 了解有关检测农药残留的方法。随后, 进一步检索每种分析方法及其在农药残留分析中的应用，阅读了近百篇相关文献，仔细对比篮选后， 反复研读了其中相关度较高的数十篇文献, 以了解每种分析方法的原理、设备、操作等信息。我们 希望选择适合本科生, 开放实验室能够提供所需仪器, 容错率、准确性相对较高的一种方法, 并进 行优化, 以供后续实验参考。

目前常用的农药残留分析方法包括: 酶抑制法、色谱法、免疫分析法、化学发光法 ${ }^{[1]}$ 和生物传感 法 ${ }^{[2,3]}$ 等, 部分主要的分析方法的特点及优劣对比列举如表 2(a)、2(b) ${ }^{[4-13]}$ 所示。

这些方法各具特点, 各有优劣, 我们应该根据情况和需求合理挑选。

如今科技发展迅猛，每种方法都在朝着操作简便、灵敏度高、应用面广、耗时短等方面改进。 除以上方法外, 新型的检测方法正不断涌现, 如 $\mathrm{Fe}_{3} \mathrm{O}_{4}$ 磁性纳米颗粒过氧化酶比色法 ${ }^{[14]}$ 、压电生物 传感器法 ${ }^{[15]}$ 等。每一种方法都有其特殊的检测对象, 以及相应的优势。如 $\mathrm{Fe}_{3} \mathrm{O}_{4}$ 磁性纳米颗粒过氧 化酶比色法不仅能够辨别是否存在农药残留, 还能快速、灵敏地评估绝大多数有机磷化合物的神经 毒性。

还有一些方法利用仪器联用技术, 例如 Perret D. 等 ${ }^{[16]}$ 利用液相色谱-质谱法测定商品果汁中的农 药残留量, 方法检出限均小于 $0.2 \mu \mathrm{g} \cdot \mathrm{kg}^{-1}$, 样品在 2-50 ng. $\mathrm{g}^{-1}$ 范围内的分析的相对标准偏差低于 $9 \%$ 。 Granby K 、 J Johanesen S. 等 ${ }^{[17]}$ 利用高效液相色谱与电喷雾电离质谱快速测定谷物中草甘膦, 在 $0.03-$ $0.33 \mathrm{mg} \cdot \mathrm{kg}^{-1}$ 的加标水平下获得 $85 \%$ 的平均回收率 $(n=32)$ 。色谱与质谱结合联用, 可以同时发挥色 谱的分离优势和质谱的鉴别优势, 避免杂质峰造成的误判。

综合考虑文献调研中提及的各种分析方法、实践队成员所学的知识以及学院开放实验室的设备 条件, 我们决定以酶抑制分光光度法为基础, 开展一系列实验研究。

表 2(a) 农药残留的分析方法特点对比

\begin{tabular}{|c|c|c|c|c|c|c|}
\hline 方法 & 灵敏度 & 设备要求 & 耗时 & 单次分析组分 & 分析过程 & 使用场合 \\
\hline 波谱法[4] & $1-10 \mathrm{ng}$ & 普通 & 短 & 单 & 简单 & 快速鉴定 \\
\hline 薄层色谱法 $\left.{ }^{4]}\right]$ & $0.1-1 \mu \mathrm{g}$ & 普通 & 长 & 多 & 复杂 & 常规分析 \\
\hline 高效薄层色谱法 ${ }^{[5]}$ & $20-300 \mathrm{ng}$ & 专业 & 较长 & 多 & 较为复杂 & 常规分析 \\
\hline 气相色谱法 ${ }^{[4]}$ & $0.05-1 \mathrm{ng}$ & 专业 & 短 & 多 & 复杂 & 常规分析 \\
\hline 毛细管电泳色谱法 ${ }^{[6]}$ & $10 \mathrm{ng}$ & 专业 & 较短 & 多 & 简单 & 常规分析 \\
\hline 高效液相色谱法 ${ }^{[7]}$ & $1 \mathrm{ng}$ & 专业 & 短 & 多 & 复杂 & 常规分析 \\
\hline 超临界流体色谱法 & $0.01 \mathrm{mg} \cdot \mathrm{kg}^{-1}$ & 专业 & 较长 & 多 & 复杂 & 常规分析 \\
\hline 紫外-可见分光光度法 & $1-10 \mu \mathrm{g}$ & 较普通 & 短 & 单 & 简单 & 快速检测 \\
\hline 表面增强拉曼光谱法 ${ }^{88]}$ & $2-5 \mathrm{ng}$ & 很专业 & 短 & 多 & 简单 & 快速检测 \\
\hline 酶抑制法 & $0.1-3 \mathrm{mg} \cdot \mathrm{kg}^{-1}$ & 较普通 & 短 & 无法分离 & 简单 & 快速鉴定 \\
\hline 免疫分析法 ${ }^{[9]}$ & $0.01-0.1 \mu \mathrm{g} \cdot \mathrm{kg}^{-1}$ & 较普通 & 短 & 单 & 简单 & 快速检测 \\
\hline
\end{tabular}


表 2(b) 农药残留的分析方法的优劣对比

\begin{tabular}{|c|c|c|}
\hline 方法 & 优势 & 劣势 \\
\hline 波谱法 & 耗时短, 操作简单, 可用于快速鉴定 & $\begin{array}{l}\text { 灵敏度不高, 实验干扰因素较多, 易出现假阴性, } \\
\text { 一次只能测定一种或一类农药 }\end{array}$ \\
\hline 薄层色谱法 & $\begin{array}{l}\text { 技术成熟, 应用面广, 操作较为简单, 可用于快速检 } \\
\text { 测, 可分离复杂混合物 }\end{array}$ & $\begin{array}{l}\text { 耗时较长, 无法定量, 重现性较差, 操作流程较为 } \\
\text { 繁琐 }\end{array}$ \\
\hline 高效薄层色谱法 & 耗时较短, 分离能力更强, 重现性较好 & 操作较为繁琐, 检测时间长, 应用面较窄 \\
\hline 气相色谱法 & $\begin{array}{l}\text { 耗时短、高选择性、仪器造价低廉、能够一次性分析 } \\
\text { 多种有机磷化合物 }\end{array}$ & 仪器造价昂贵, 操作较为繁琐, 不能直接定性 \\
\hline 毛细管电泳色谱法 & 耗时短, 可分离复杂混合物[10] & 易产生组分改变, 重现性较差, 灵敏度较低 \\
\hline 高效液相色谱法 & 能够测定沸点高、热稳定性差、极性强的化合物 & 仪器造价昂贵, 准备工作复杂, 检测时间长 ${ }^{[11]}$ \\
\hline 超临界流体色谱法 & 可分离量大、热不稳定的化合物, 分离能力较强 ${ }^{[2]}$ & 操作难度大, 检测时间长, 应用面窄 ${ }^{[11]}$ \\
\hline $\begin{array}{l}\text { 紫外-可见分光光度法 } \\
\text { 表面增强拉曼光谱法 }\end{array}$ & $\begin{array}{l}\text { 耗时短, 应用面较广, 操作简单, 可用于快速检测 } \\
\text { 耗时短, 操作简单, 能够提供丰富的化合物结构信 } \\
\text { 息, 可用于快速检测 }\end{array}$ & $\begin{array}{l}\text { 灵敏度不高, 干扰因素多, 易出现假阴性 } \\
\text { 仪器造价昂贵 }\end{array}$ \\
\hline 酶抑制法 & 耗时短, 操作简单, 可用于快速鉴定 & 反应条件难以把握, 只能给出定性结果 \\
\hline 免疫分析法 & $\begin{array}{l}\text { 检测快速, 样品前处理简单, 灵敏度高, 特异性高, } \\
\text { 可用于快速检测 }\end{array}$ & 原料制备复杂，开发成本较高 ${ }^{[13]}$ \\
\hline
\end{tabular}

\section{3 实验}

有机磷农药是种类最繁多的农药之一，如敌敌畏、乐果、敌百虫等。其在农业生产中的广泛 应用, 可能导致不同程度的农药残留。有机磷农药挥发性强, 微溶于水, 遇碱不稳定, 可能对人 体造成急性中毒 ${ }^{[19]}$, 大剂量接触会造成呼吸麻痹, 甚至死亡。鉴于以上特点, 我们选用其作为本 次实验使用的农药。

酶抑制分光光度法是较早成型的、操作最为简单的方法之一, 所以被广泛应用于农药残留检 测 ${ }^{[18]}$ 。国家标准 GB/T 18630-2002 正是利用酶抑制分光光度法进行快速检测蔬菜中农药残留的一种 常用且简便的方法。

虽说酶抑制分光光度法有诸多优势, 我们也考虑到该方法存在的不足之处: 首先, 不同农药对 酶的抑制效应不同, 实际检测中使用的酶抑制分光光度法只能给出定性的检验结果, 且具有较大的 不确定度; 其次不同来源、不同方法制备的酶催化活性不同, 可能造成误差; 再次, 酶与底物混合 后在确定的相同的反应时间下检测的要求也导致无法同时处理多份样品(包括无法同时进行加热), 大大降低了分析效率。

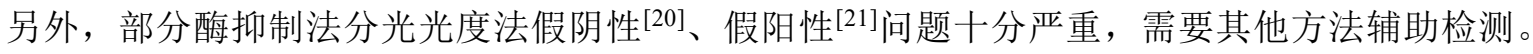
因而, 我们希望在实验过程中对实验方法进行优化，从而发挥酶抑制分光光度法在便捷性上的优势， 而解决其不足之处, 从而使该方法更加适用于农药残留的快速检测和大批量检测。

\section{1 仪器和试剂}

仪器：岛津 UV-3600Plus 和 cary60 紫外-可见分光光度计。

试剂: 五种有机磷农药的混合丙酮溶液 (敌敌畏 $103 \mu \mathrm{g} \cdot \mathrm{mL}^{-1}$, 乐果 $104 \mu \mathrm{g} \cdot \mathrm{mL}^{-1}$, 对硫磷 $99.4 \mu \mathrm{g} \cdot \mathrm{mL}^{-1}$, 马拉硫磷 $102 \mu \mathrm{g} \cdot \mathrm{mL}^{-1}$, 毒死蜱 $99.2 \mu \mathrm{g} \cdot \mathrm{mL}^{-1}$, 扩展不确定度 $5 \%, k=2$, 北京海岸鸿 蒙标准物质技术有限责任公司); 乙酰胆碱酯酶液 $(2 \%$, 溶于 $\mathrm{pH}=7.71$ 缓冲溶液, 海荭兴仪器股份有 
限公司); 2,6 -二氯靛酚钠二水合物( $95.0 \%$, 国药集团化学试剂有限公司); 碘化乙酰硫代胆碱( $>98 \%$, 生物试剂, 国药集团化学试剂有限公司); 磷酸氢二钠、磷酸二氢钠、丙酮、碳酸钙、活性炭均为分 析纯试剂。

\section{2 酶抑制分光光度法的原理}

乙酰胆碱酯酶( $\mathrm{AChE}$ )能够催化乙酰胆碱水解为胆碱和乙酸 ${ }^{[22]}$ 。其反应机理为: 1) 乙酰胆碱的季 铵盐基团与乙酰胆碱酯酶的芳香族氨基酸(如色氨酸、酪氨酸)通过静电作用结合，形成 Michaelis 酶-底物络合体, 使乙酰胆碱酯酶的丝氨酸活性中心与乙酰胆碱的酯基处于合适的空间位置; 2) 丝 氨酸的差基在旁侧组氨酸的咪唑基氮诱导下对乙酰胆碱的酯基发生亲核取代反应, 乙酰基转移到乙 酰胆碱酯酶上, 形成乙酰化乙酰胆碱酯酶; 3) 乙酰化乙酰胆碱酯酶不稳定, 在组氨酸诱导下以相似 的亲核取代机理迅速水解使酶复活(图 6)。
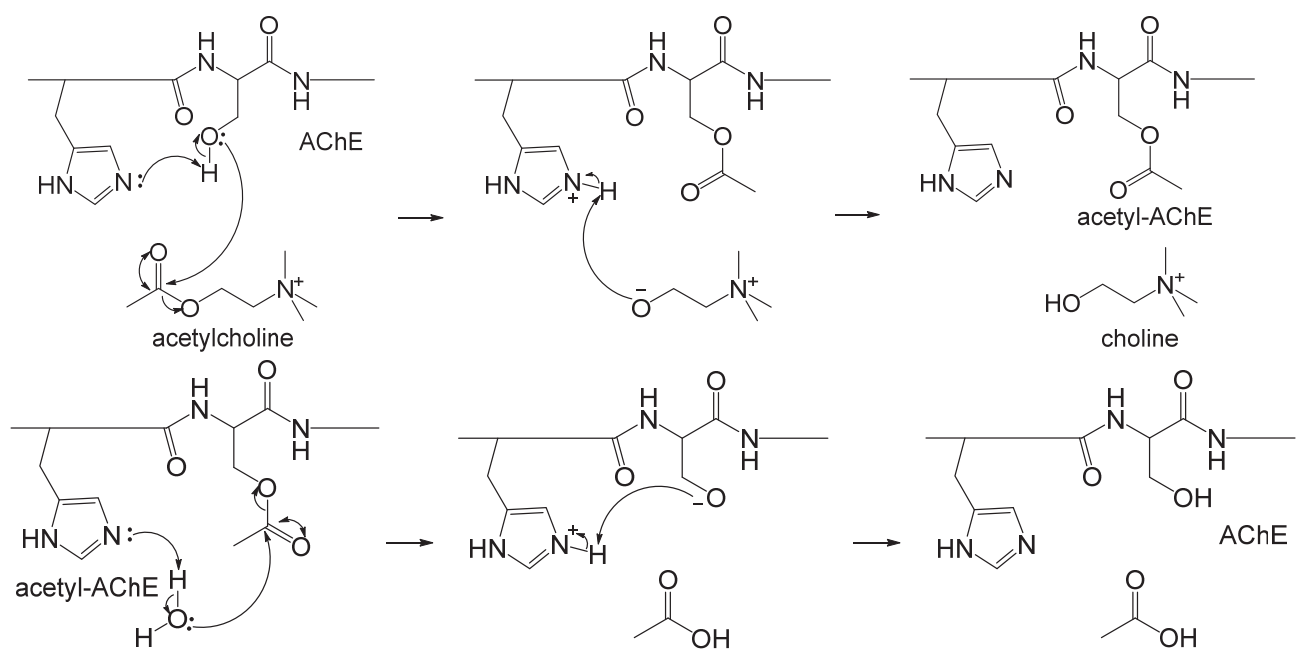

图 6 乙酰胆碱酯酶催化乙酰胆碱水解机理

由于乙酰胆碱酯酶是一广谱的催化剂, 除催化乙酰胆碱及其硫代物等衍生物的水解反应外, 还 可催化芳香酯、酰基苯胺、酰胺、硫代酯等乙酰胆碱类似物的水解反应 ${ }^{[22]}$ 。而有机磷农药多为磷酸 酯或硫代磷酸酯(图 7), 含有磷酸酯基或硫代磷酸酯基。

天然底物乙酰胆碱含有酯基, 二者在结构上具有相似性, 因而有机磷农药与乙酰胆碱一样可以 与乙酰胆碱酯酶的活性中心有效结合, 且有机磷化合物与酶的反应速率高于天然底物乙酰胆碱。

但有机磷化合物与乙酰胆碱酯酶反应所得的产物磷酰化乙酰胆碱酯酶相对稳定, 不易水解, 因 而无法使酶恢复活性，从而实现了对酶促反应的抑制。其抑制机理属于竞争性抑制[23]，如图 8 所示。

在酶源、温度、反应时间等其他条件均相同时, 产物的浓度与有机磷化合物的浓度具有单调函 数关系, 通过显色反应分别测定样品与无农药对照组的产物的浓度, 即可计算出样品的抑制率, 通 过抑制率标准曲线可获知有机磷化合物的浓度。

\section{3 实验内容}

\subsection{1 条件实验}

为排除仪器差异对测量值的影响, 本实验全程使用同一台分光光度计, 因而对一组多份样品只 能逐一测定。若要保证所有样品测定时温度相同, 后测定的样品势必需要恒温更长时间; 而若要保 证恒温时间相同, 后测定的样品在等待测定的过程中则会逐渐降温。即对于同一批次不同样品的测 试, 水浴恒温的时间与测定时样品的温度这两个变量无法同时控制。为了得到更加可靠的结果, 需 要通过预实验探究这两个因素对酶活性的影响情况。 
为使实验结果更好地反映测定实际样品时的情况, 我们分析了国家标准列出的各种蔬菜中允许 残留农药最大值, 并取平均值 $0.05 \mu \mathrm{g} \cdot \mathrm{mL}^{-1}$ 作为拟定的 “超标限” 。考虑到实际样品测定中农药浓 度一般低于超标限, 故用 $0.005 \mu \mathrm{g} \cdot \mathrm{mL}^{-1}$ (超标限的 0.1 倍)的溶液进行预实验。<smiles>CCOP(=S)(OCC)Oc1ccc([N+](=O)[O-])cc1</smiles>

parathion 对硫磷<smiles>CNC(=O)CSP(=O)(OC)OC</smiles>

omethoate 氧化乐果<smiles>COP(=O)(OC)OC=C(Cl)Cl</smiles>

DDVP 敌敌畏<smiles>CCSCCOP(=S)(OC)OC</smiles>

demeton methyl 甲基内吸磷<smiles>CCOP(=S)(OCC)Oc1nc(Cl)c(Cl)cc1Cl</smiles>

chlorpyrifos 氯吡硫磷(毒死蜱)<smiles>CNC(=O)CSP(=S)(OC)OC</smiles>

dimethoate 乐果<smiles>COP(=O)(OC)OC(Br)C(Cl)(Cl)Br</smiles>

dibrom 二溴磷<smiles>CCOP(=S)(OCC)SCSCC</smiles>

thimet 甲拌磷<smiles>CC(=O)OCC[N+](C)(C)C</smiles>

图 7 部分常见有机磷农药及乙酰胆碱的结构式

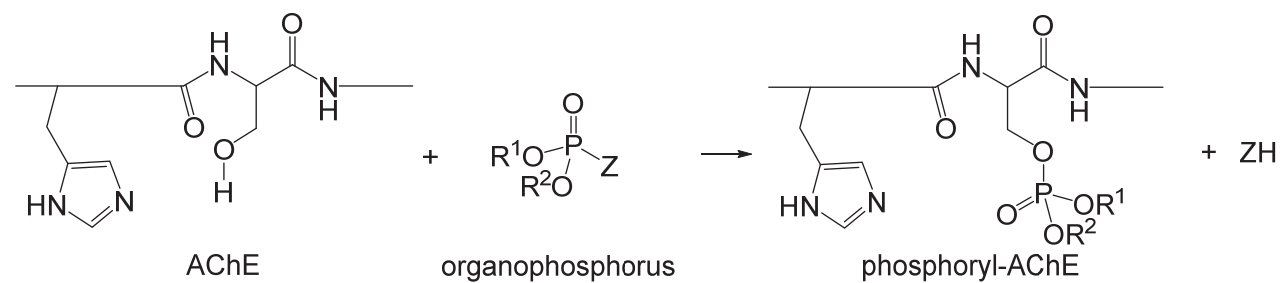

图 8 有机磷农药与乙酰胆碱酯酶的反应

通常采取的实验方案要求将测试液在 $37^{\circ} \mathrm{C}$ 下恒温 $15 \mathrm{~min}$, 随后进行测定。为探究恒温时间对酶 活性的影响, 首先取 10 组浓度为 $0.005 \mu \mathrm{g} \cdot \mathrm{mL}^{-1}$ 的农药溶液, 加入酶与显色剂后, 在 $37^{\circ} \mathrm{C}$ 水浴中分 别恒温 10-19 $\mathrm{min}$, 随后加入底物, 测定加入后每 $30 \mathrm{~s}$ 时溶液的吸光度, 得到恒温时间为 10-19 $\mathrm{min}$ 的测量结果(图 9)。

由图 9 可见, 恒温时间在 10-19 min 内时, 吸光度与恒温时间无显著相关性, 数据波动属正常 
的仪器误差。由此, 我们推测要求恒温 $15 \mathrm{~min}$ 的目的在于使酶与农药的反应达到平衡。因而, 实际 实验中只需保证样品在 $37^{\circ} \mathrm{C}$ 下保持一段时间，而不必精确控制各组样品恒温时间完全相同。

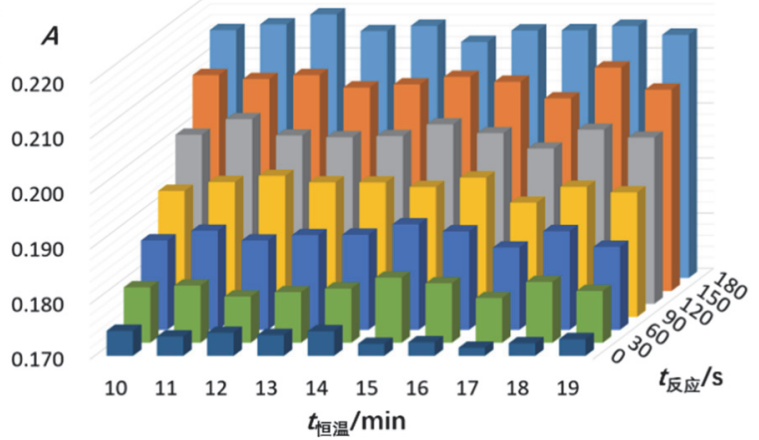

图 9 农药溶液 $37^{\circ} \mathrm{C}$ 恒温不同时间后加入底物，其吸光度值随时间的变化情况

为探究温度对酶活性的影响, 进行了以下两组实验。第一组, 取 4 份浓度为 $0.005 \mu \mathrm{g} \cdot \mathrm{mL}^{-1}$ 的农 药溶液, 加入酶与显色剂后, 在 $37^{\circ} \mathrm{C}$ 水浴中恒温 $10 \mathrm{~min}$, 取出第一份样品, 加入底物反应, 每 $30 \mathrm{~s}$ 测定一次溶液的吸光度, 其他样品仍在水浴锅中恒温, 时间满 $3 \mathrm{~min}$ 立即取出下一份样品, 进行相 同的操作; 第二组, 取 4 份浓度为 $0.005 \mu \mathrm{g} \cdot \mathrm{mL}^{-1}$ 的农药溶液, 加入酶与显色剂后, 在 $37^{\circ} \mathrm{C}$ 水浴中 恒温 $10 \mathrm{~min}$, 取出所有样品, 逐一测定每一份样品, 后测的样品均处于自然降温过程。

即第一组每份样品恒温后立即取出测量, 因此测量时温度保持恒定, 但恒温时间不同; 第二组 恒温时间相同, 但由于测量需要耗时, 每份样品测量前自然降温程度不同, 因而测试时温度不同。 根据两组实验数据，求算各时刻下吸光度相对 0 时刻的增长率，如图 10 所示。

测定结果表明, 第一组测定前始终保持恒温, 先后测定的样品吸光度增长率变化不大(图 10(a)); 而第二组在无保温的情况下, 后测定的样品由于温度降低, 吸光度增长率显著降低(图 10(b))。根据 本实验的原理, 测定的吸光度与酶促反应产物浓度正相关, 由于该反应对底物是零级反应, 吸光度 增长率反映了酶的活性, 因而, 温度对酶活性有较大影响。

综合考虑上述两组实验结果, 恒温时间对酶活性无显著影响, 而温度对酶活性有显著影响, 因 而在后续实验时, 将所有未测定样品均置于 $37^{\circ} \mathrm{C}$ 水浴中保温, 直至测定开始。

(a)

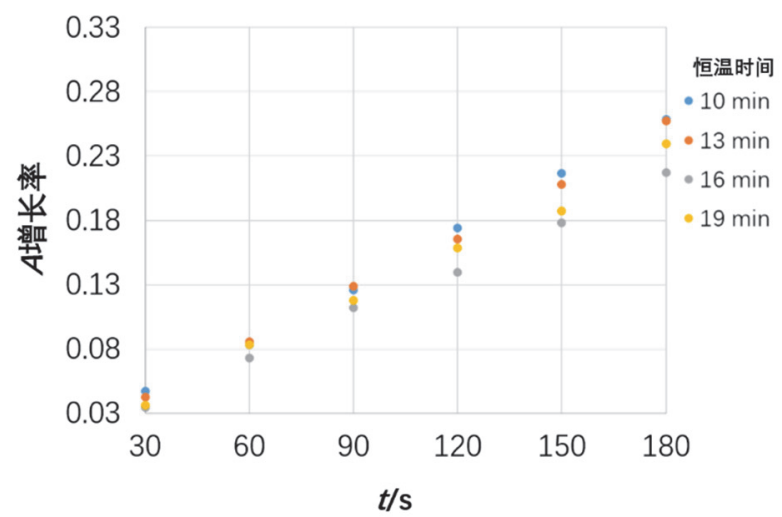

(b)

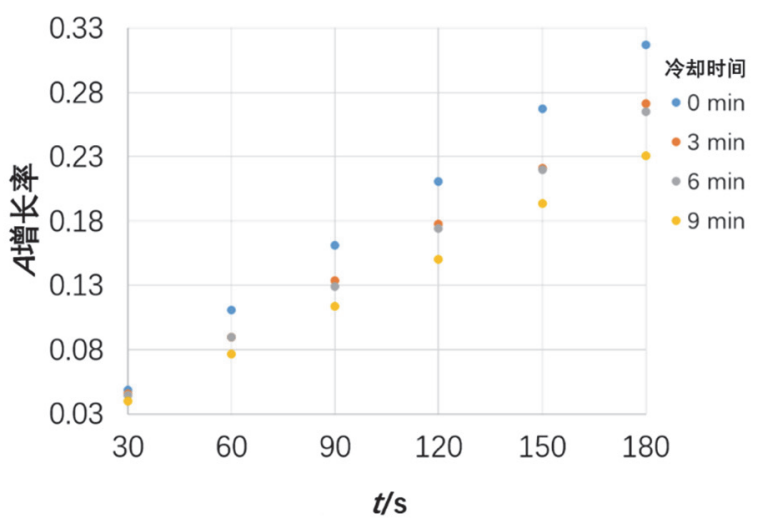

图 10 （a）农药溶液 $37^{\circ} \mathrm{C}$ 恒温不同时间后加入底物, 溶液吸光度值随反应时间的相对增长率; (b) 农药溶液 $37^{\circ} \mathrm{C}$ 恒温 $10 \mathrm{~min}$ 后自然冷却不同时间再加入底物, 溶液吸光度值随反应时间的相对增长率 


\subsection{2 方法验证}

本实验中采用海荭兴仪器股份有限公司提供的检测试剂盒, 包括: $2 \%$ 乙酰胆碱酶液、 $\mathrm{pH}=7.71$ 的缓冲溶液、 $2 \%$ 碘化硫代乙酰胆碱底物溶液、去离子水及显色剂。

根据检测试剂盒说明书, 需要测定加入底物后瞬时的溶液吸光度 $A_{0}$ 和反应时间为 $1 \mathrm{~min}$ 时的吸 光度 $A_{t}$, 并根据式(1)计算反应时间为 $1 \mathrm{~min}$ 时的抑制率 $R$ :

$$
R=\frac{A_{t}-A_{0}}{A_{0}}
$$

其中, $A_{0}$ 为反应时间为 0 的吸光度值, $A_{t}$ 为给定反应时间 $t$ 下的吸光度值。抑制率反映了产物的生 成量, 相同时间下的抑制率即可反映酶促反应的速率, 从而反映酶活性受到抑制的程度, 最终反映 农药的浓度。抑制率越高, 表明样品中农药浓度越高。

然而 0 时刻的吸光度 $A_{0}$ 在实际操作过程中是无法准确测定的, 混合均匀底物与溶液并倒入比色 皿进行测定需要花费一定的时间，且该时间在各次测量过程中不同，会造成较大的误差。

根据化学反应动力学知识, 我们考虑到酶促反应中酶的浓度不足, 而底物大大过量, 因而酶促 反应对底物为一级反应, 反应产物的浓度应当与时间成线性关系。而产物随后的显色反应相较酶促 反应为快平衡反应, 因而溶液吸光度亦应与时间成线性关系。如果能证明这一关系, 我们便能够通 过测定一系列反应时间下的吸光度，线性回归外推出 0 时刻准确的吸光度值。

为验证这一猜想, 我们配制了一系列已知浓度的农药标准溶液, 分别取各浓度标准溶液 $3 \mathrm{~mL}$, 预先加入 $100 \mu \mathrm{L}$ 酶液、 $100 \mu \mathrm{L}$ 显色剂并于 $37^{\circ} \mathrm{C}$ 水浴中恒温 $10 \mathrm{~min}$, 随后加入底物并同时开始计 时, 自 $30 \mathrm{~s}$ 起每隔 $30 \mathrm{~s}$ 测定溶液的吸光度值(表 3), 并作图(图 11)。

表 3 不同浓度的系列农药标准溶液在反应开始后每隔 $30 \mathrm{~s}$ 的吸光度

\begin{tabular}{ccccccccc}
\hline \multirow{2}{*}{ 农药标液浓度 $c /\left(\mu \mathrm{g} \cdot \mathrm{mL}^{-1}\right)$} & \multicolumn{5}{c}{ 不同反应时间下的吸光度 $A$} \\
\cline { 2 - 8 } & $0 \mathrm{~s}$ & $30 \mathrm{~s}$ & $60 \mathrm{~s}$ & $90 \mathrm{~s}$ & $120 \mathrm{~s}$ & $150 \mathrm{~s}$ & $180 \mathrm{~s}$ \\
\hline 0 & 0.182 & 0.191 & 0.199 & 0.204 & 0.214 & 0.222 & 0.231 \\
$5.00 \times 10^{-4}$ & 0.183 & 0.189 & 0.197 & 0.203 & 0.210 & 0.218 & 0.226 \\
$2.50 \times 10^{-3}$ & 0.182 & 0.189 & 0.195 & 0.200 & 0.206 & 0.213 & 0.220 \\
$5.00 \times 10^{-3}$ & 0.182 & 0.188 & 0.193 & 0.198 & 0.204 & 0.209 & 0.215 \\
0.0500 & 0.184 & 0.188 & 0.192 & 0.196 & 0.200 & 0.204 & 0.209 \\
0.125 & 0.183 & 0.187 & 0.191 & 0.194 & 0.197 & 0.203 & 0.205 \\
0.250 & 0.183 & 0.186 & 0.189 & 0.192 & 0.195 & 0.198 & 0.202 \\
0.375 & 0.183 & 0.186 & 0.188 & 0.191 & 0.193 & 0.196 & 0.199 \\
0.500 & 0.183 & 0.185 & 0.187 & 0.189 & 0.192 & 0.194 & 0.197 \\
1.25 & 0.183 & 0.186 & 0.187 & 0.189 & 0.192 & 0.193 & 0.195 \\
\hline
\end{tabular}

其中 0 时刻吸光度值为线性回归外推求得

由图 11 可见, 各浓度的标准溶液其吸光度均与反应时间呈良好的线性关系。这证明了我们通过 测定系列时间下的吸光度从而外推出 0 时刻吸光度, 并准确计算反应时间为 $1 \mathrm{~min}$ 时的抑制率的方 法是可行的。

根据表 1 的数据按式(1)计算反应时间为 $1 \mathrm{~min}$ 时的抑制率 $R$, 所得结果见表 4 。从表 4 可见, 农药浓度在 $0.005 \mu \mathrm{g} \cdot \mathrm{mL}^{-1}$ 以上的抑制率均大于 $50 \%$, 这一结果与快速检测试剂盒说明书给出的 “抑 制率大于 $50 \%$ 判定为超标样品” 的判据吻合度很高, 从而证实了该方法的可靠性。因此在随后的实 际样品测定中，我们采用了上述方法。 


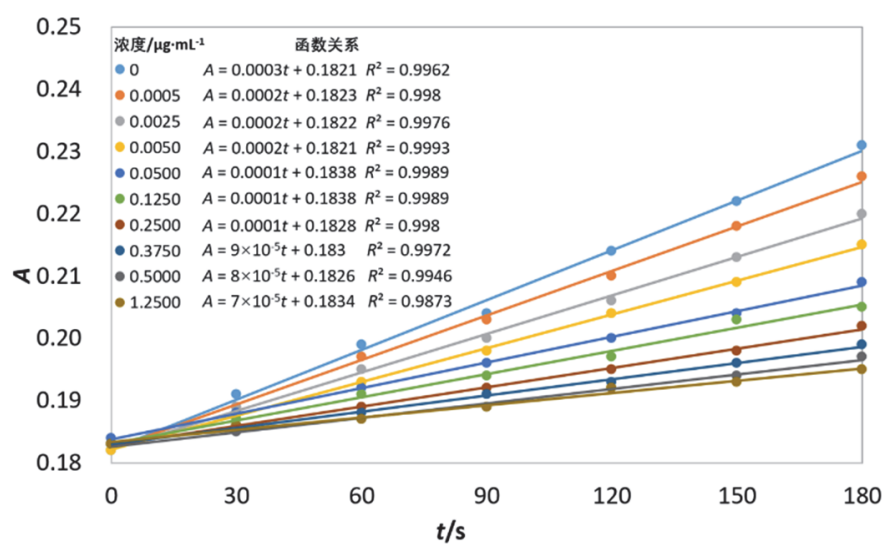

图 11 溶液吸光度与反应时间的关系

表 4 反应时间为 $1 \mathrm{~min}$ 时各农药标准溶液对应的抑制率

\begin{tabular}{cc}
\hline 农药浓度 $/\left(\mu \mathrm{g} \cdot \mathrm{mL}^{-1}\right)$ & $R / \%$ \\
\hline 0 & 16.3 \\
$5.00 \times 10^{-4}$ & 22.3 \\
$2.50 \times 10^{-3}$ & 35.8 \\
$5.00 \times 10^{-3}$ & 50.2 \\
0.0500 & 53.8 \\
0.125 & 62.2 \\
0.250 & 70.5 \\
0.375 & 75.7 \\
0.500 & 76.5 \\
\hline
\end{tabular}

\subsection{3 标准样品检测试纸快速检测}

我们在社会实践调查中关注到农药残留检测试纸的问题, 我们想通过实际实验检验其是否能准 确检测出农药残留。于是我们购买了由海荭兴仪器股份有限公司生产的农药残留速测卡, 此检测卡 依照国家标准方法 GB/TB5009.199-2003，原理采用酶抑制法。

我们配置了 $0.00 、 0.05 、 0.10 、 0.20 、 0.40 、 0.60 、 0.80 \mu \mathrm{g} \cdot \mathrm{mL}^{-1}$ 共七份农药样品, 分别取 3 滴到 白色区域(速测卡上部), 在 $37^{\circ} \mathrm{C}$ 恒温装置放置 $10 \mathrm{~min}$, 随后对折速测卡, $3 \mathrm{~min}$ 后观测实验结果如 图 12 所示。

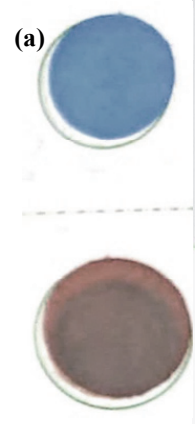

(b) (c)
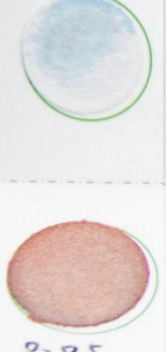

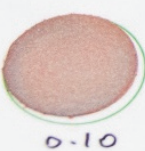

(d)

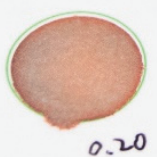

(e)

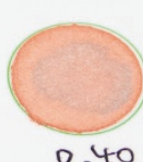

(f)

(g)

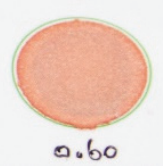

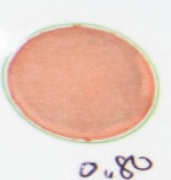

图 12 检测不同浓度的农药后的速测卡

使用农药含量分别为：(a) 对照组; (b) $0.05 \mu \mathrm{g} \cdot \mathrm{mL}^{-1}$; (c) $0.10 \mu \mathrm{g} \cdot \mathrm{mL}^{-1}$; (d) $0.20 \mu \mathrm{g} \cdot \mathrm{mL}^{-1}$;

(e) $0.40 \mu \mathrm{g} \cdot \mathrm{mL}^{-1}$; (f) $0.60 \mu \mathrm{g} \cdot \mathrm{mL}^{-1}$; (g) $0.80 \mu \mathrm{g} \cdot \mathrm{mL}^{-1}$

电子版为彩图 
如果白色部分变为深蓝色, 如图 12(a), 表示为阴性结果。如果含有较低浓度农药, 原白色部分 显浅蓝色, 如图 12(b)、(c)、(d); 含较高浓度白色部分不变色, 如图 12 的(e)、(f)、(g)。我们发现农 药浓度在 $0.05 \mu \mathrm{g} \cdot \mathrm{mL}^{-1}$ 时, 蓝色已经比较微弱, $0.40 \mu \mathrm{g} \cdot \mathrm{mL}^{-1}$ 左右白色部分便不再变色。按照 $\mathrm{GB} 2763-$ 2019 食品中农药最大残留限量国家标准, 此检出限已经符合对应农药的最低标准, 所以使用该速测 卡是能够比较有效地检测出超标农药残留。

\subsection{4 实际样品测定}

我们分别在超市和菜市场采购了一批蔬菜样品, 包含卷心菜、上海青、芹菜等, 用于实际样品 的测定。

测定过程中, 我们将待测各种蔬菜剪为 $1 \mathrm{~cm}$ 见方大小, 使其均匀, 称重后确定缩分次数, 用四 分法取出大于 $10 \mathrm{~g}$ 的最少量样品, 从中用天平称出两份 $5 \mathrm{~g}$ 的样品, 分别加入 $10 \mathrm{~mL}$ 缓冲溶液, 振 荡 1-2 min, 并静置 $2 \mathrm{~min}$, 随后过滤得提取液。分别取每份提取液 $3 \mathrm{~mL}$ 于独立的试管中, 并取 $3 \mathrm{~mL}$ 缓冲溶液于另一试管中, 分别加入 $100 \mu \mathrm{L} 2 \%$ 酶液, $100 \mu \mathrm{L}$ 显色剂溶液, 震荡均匀后置入 $37^{\circ} \mathrm{C}$ 恒温水浴锅中, $10 \mathrm{~min}$ 后开始逐一取出样品提取液测定。

测定时, 先向盛有样品提取液的试管中加入 $100 \mu \mathrm{L} 2 \%$ 碘化硫代乙酰胆碱(底物)溶液, 立即震荡 均匀, 同时开始计时, 自 $30 \mathrm{~s}$ 起至 $180 \mathrm{~s}$ 每隔 $30 \mathrm{~s}$ 测定一次吸光度, 并外推出 0 时刻吸光度值, 按 式(1)计算抑制率。

测定结果显示, 我们采购的所有样品均小于 $25 \%$, 表明无农药残留超标的情况。

为了验证实验结果, 我们也采取了速测卡检测实际样品, 结果同样显示所有样品均无农药残留 超标的情况。这也从一个侧面反映出, 超市和菜市场的蔬菜合格率还是比较高的。

\section{4 清除蔬菜农药残留的方法探究}

我们根据社会调研的结果拟定了不同的清除农药残留的方法, 由于 3.3 .4 节的检测中没有发现 农药残留超标的蔬菜, 所以我们使用经相同的农药处理后的样品模拟农药残留超标样品, 以探究清 除农药残留的各种方法的有效性。

无法避免的是, 本实验中农药喷酒后放置的时间相较于真实情况短。而且实践活动中的实验重 复次数不足, 只能给出相应的建议。

模拟样品在使用不同方法清除农药残留后, 按照 3.3.3 节中的方法进行样品处理和测定。我们分 别考察了不同时间的水浸泡法、碱水法、盐水法、加热法等处理方法, 并分别对抑制率进行计算, 并设立一个不做处理的对照组作为对比组, 所得结果如图 13 所示。从图 13 我们可以看出, 所有的 缓水清洗、自来水浸泡、盐水浸泡、蔬菜清洗剂洗涤、醋浸泡组的酶抑制率都在对照组的波动范围 之内, 即我们认为这些方法对农药残留的去除效果有限, 没有显著的去除效果。热水与碱浸泡组的 抑制率要明显低于其他组，我们认为这两种处理方法比其他方法具有更好的洗去农药的效果。

显然, 我们在社会问卷中得到的部分处理方法, 如用盐水浸泡、用醋浸泡并没有很显著的效果。

我们采用的农药为有机磷类、氨基甲酸酯类的五种农药。有机磷类杀虫剂难溶于水, 所以用清 洗和浸泡的方法只能除去部分的农药。但是考虑到水洗法是去除农药残留的基础方法, 所以建议先 冲洗, 冲洗时间控制在 15-30 s; 再浸泡, 浸泡时间控制在 5-10 min, 因为浸泡时间过长时, 死亡的 蔬菜细胞由于失去膜的选择透过性, 会重新吸收农药。

由于蔬菜清洗剂中的表面活性剂可以增加农药在水中的溶解度, 所以, 适当加入蔬菜清洗剂有 助于去除农药。随着温度升高, 大部分农药会开始分解, 并且温度越高, 分解速度越快, 所以也可 以将蔬菜浸入热水中达到除去农药残留的目的。同样的, 我们不能在热水中浸泡过久, 否则蔬菜亦 会重新吸收农药, 一般浸泡时间控制在 10-15 min 为宜。 


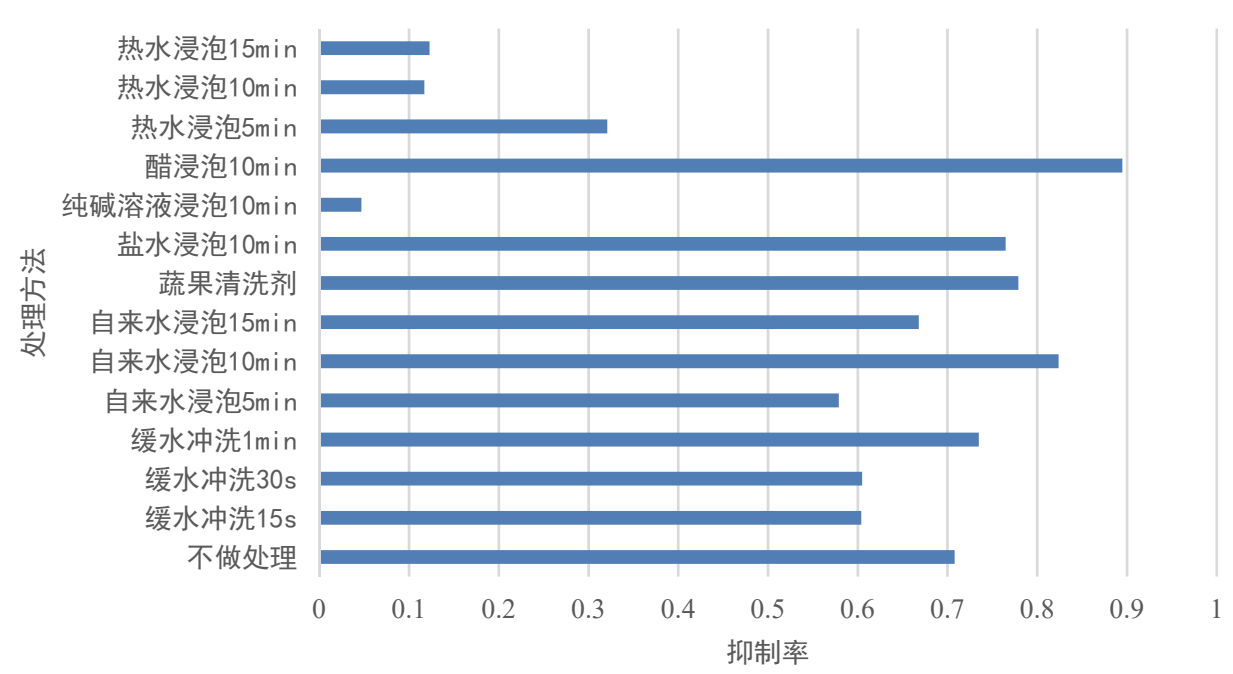

图 13 不同处理方法后的酶抑制率

由于有机磷类农药和氨基甲酸酯类农药在碱性条件下分解, 所以碱水浸泡法可以很有效地去除 农药残留, 是最推荐使用的方法。而盐水和醋处理, 实际上与用水处理无显著区别, 不推荐使用。 醋甚至会抑制农药的水解, 因而醋处理是最不推荐的处理方法。

综上所述, 我们建议消费者主要采取冲洗再浸泡、辅助使用盐、蔬果清洗剂之类的方法, 能够 较为有效地实现农残去除。

\section{5 结语}

从前期准备到正式活动, 整个实践活动费时约 2 个月, 后期的活动进展比较顺利, 是因为我们 实践队在初期便确定了三个分组：社会实践组、原理实验组和探究设计组。

社会实践组以自身经历和经验为基础, 通过调查问卷、现场采访收集到大家对农药残留的态度 以及处理农药残留的方式, 并在企业调研中了解到现在前沿的农药残留检测方法; 原理实验组依照 企业调研结果、收集到的文献以及学院开放实验室所提供的条件等, 寻找最适合我们实践活动的方 法; 探究设计组根据社会实践组调查问卷总结的市民常用的农药残留处理方法, 再依据原理实验组 提供的方法对其进行可行性分析。每一个小组分工明确, 环环相扣, 使得实践进展快速、高效。

本次实践活动, 通过不同范围的采访和调查, 我们体会到向市民科普农药残留知识的重要性, 了解到发展市场接受的快速检测方法的迫切需求及其所处的困境。如果想要改善上述现象, 仍需有 更加积极的宣传对策和更加先进的检测方案。

同样, 我们知道由于在实际应用中农药种类未知, 利用酶抑制法通常无法得出定量的分析结果, 只能给出定性的结论。我们实验的系列标准样品由质量体积浓度为 $100 \mu \mathrm{g} \cdot \mathrm{mL}^{-1}$ 的五种确定农药的 丙酮溶液稀释而得, 因此也只能在相对应的体系中具有准确定量的分析结果。若需推广至实际应用, 仍需定量分析不同农药对该酶抑制法的抑制作用差异，寻求合理的标准方程。

在此次社会实践活动中，我们也遇到了一些困难。

调查问卷的设计是我们最早遇到的难题, 社会实践组为此更改过近 20 次, 为的是既能做到通俗 易懂、结构合理、逻辑性强, 又能使每一个问题都有其意义所在。

原理实验组在确定实验方案前查阅了大量相关的中英文文献, 通过实验求证, 再根据现实情况 做出合理的优化。在阅读对比文献的过程中, 该组同学发现检索到的文献质量参差不齐, 部分文献 存在较为明显的错误, 有些文献缺乏实际操作的可行性。这些都对最终确定农药检测的实验方案有 着比较大的影响。 
探究设计组面临最大的问题便是真实样品的检测, 他们需要在面对不同的检测样本时对原理实 验组给出的方案做出部分改动, 以求获得最佳实验结果。

不经一番寒彻骨, 怎得梅花扑鼻香。对未知的探索必然充斥着艰难困苦, 但只要我们有执着的 信念、扎实的知识和无畏的勇气, 必然能品尝到成功的硕果。最后, 十分感谢厦门大学化学化工学 院老师们的帮助。“一点一滴” 实践队的目的便是将化学运用于生活中的点点滴滴, 希望我们的实践 能对大家有所帮助。

\section{参 考 文 献}

[1] Liang, H. C.; Bilon, N.; Hay, M. T. Water Environ. Res. 2015, 87 (10), 1923.

[2] Rainina, E. I.; Efremenco, E. N.; Varfolomeyev, S. D.; Simonian, A. L.; Wild, J. R. Biosens. Bioelectron. 1996,11 (10), 991.

[3] Rogers, K. R.; Wang, Y.; Mulchandani, A.; Mulchandani, P.; Chen, W. Biotechnol. Prog. 1999, 15 (3), 517.

[4] 蔡建荣, 张东升, 赵晓联. 中国卫生检验杂志, 2002, 12 (6), 750.

[5] 樊玮, 汤锋, 岳永德. 分析仪器, 2010, No. 2, 36 .

[6] 楚清脆, 耿成怀, 林永, 叶建农. 理化检验, 2009, 45, 114.

[7] Yang, S. S.; Goldsmith, A. I.; Smetena, I. J. Chromatogr. A 1996, 754 (1-2), 3.

[8] 应方, 梁苗苗, 李剑锋. 光散射学报, 2019, 31 (2), 131.

[9] Cho, Y. A.; Seok, J. -A.; Lee, H. -S.; Kim, Y. J.; Park, Y. C.; Lee, Y. T. Anal. Chim. Acta 2004, 522 (2), 215.

[10] 林振宇, 黄露, 陈国南. 色谱, 2009, No. 1, 16 .

[11] 肖敏. 农业技术与装备, 2019, No. 4, 27.

[12] 张曙明, 陈建民, 顾志平, 赵扬. 分析试验室, 1994, 13(5), 72.

[13] 田子华. 酶抑制法农药残留快速检测技术研究[D]. 南京: 南京农业大学, 2005.

[14] Liang, M.; Fan, K.; Pan, Y.; Jiang, H.; Wang, F.; Yang, D.; Lu, D.; Feng, J.; Zhao, J.; Yang, L.; et al. Anal. Chem. 2013,85 (1), 308.

[15] Abad, J. M.; Pariente, F.; Hernández, L.; Abruña, H. D.; Lorenzo, E. Anal. Chem. 1998, 70 (14), 2848.

[16] Perret, D.; Gentili, A.; Marchese, S.; Sergi, M.; D’ascenzo, G. J. AOAC Int. 2002, 85 (3), 724.

[17] Granby, K.; Johannesen, S.; Vahl, M. Food Addit. Contam. 2003, 20 (8), 692.

[18] 刘永杰, 张金振, 曹明章, 沈晋良. 现代农药, 2004, 3 (2), 25.

[19] Mileson, B. E.; Chambers, J. E.; Chen, W. L.; Dettbarn, W.; Ehrich, M.; Eldefrawi, A. T.; Gaylor, D. W.; Hamernik, K.; Hodgson, E.; Karczmar, A. G.; et al. Toxicol. Sci. 1998, 41 (1), 8.

[20] 张瑜, 魏维杰, 李岗, 魏云潇, 邵康群. 中国卫生检验杂志, 2018, $28(14), 1760$.

[21] 杨东鹏，张春荣，董民，杜相革. 中国农学通报, 2004, 20 (2), 37.

[22] 朱美财. 生物化学与生物物理进展, 1992, 19 (5), 338 .

[23] 闵红. 蔬菜类食品中农药残留的快速检测方法和仪器的研究[D]. 上海: 华东师范大学, 2008 . 